\title{
EDITORIAL
}

\section{QRS Duration in the Selection of Patients for Cardiac Resynchronization Therapy}

\author{
Arthur J. Moss, M.D. \\ From the Cardiology Unit, Department of Medicine, University of Rochester School of Medicine and Dentistry, \\ Rochester, New York, NY
}

It has been known since the discovery of the electrocardiogram that an intraventricular conduction disturbance as manifest by widened, upright QRS complexes in leads $I$ and $V_{6}$ was associated with heart disease and increased cardiac morbidity and mortality. A left bundle branch block pattern almost always occurred in the setting of cardiac disease. In the 1950s, left-sided conduction disturbances were more clearly delineated with identification of hemiblocks involving the superior or inferior divisions of the left bundle. These hemiblocks were associated with modest prolongation of the QRS interval involving mostly the terminal portion of the QRS complex and alteration of the QRS axis.

Transvenous right ventricular pacing was introduced in the 1960s for the treatment of heart block, and pacing from the apex of the right ventricle produced a classical left bundle branch block pattern. Although the etiology of heart block could have many causes, a majority of the early group of patients requiring pacemaker therapy had heart block due to degeneration of the cardiac conduction system, so-called trifascicular block, with relatively well-preserved myocardial contractile function. The potential deleterious effects of chronic right ventricular pacing with left bundle branch block conduction disturbance were not appreciated at that time. In the late 1970s, dual-chamber transvenous pacing of the atrium and ventricle was introduced with the potential to shorten the paced PR interval thereby optimizing ventricular filling with the so-called atrial kick mechanism. It was thought that dual-chamber pacing with opti- mization of the PR interval would improve ventricular function in cardiac patients with compromised cardiac contractility and associated PR interval prolongation. In fact, the Dual-Chamber and VVI Implantable Defibrillator (DAVID) Trial was initiated in 2000 to determine if dual-chamber (DDDR) pacing with optimization of the PR interval to about $140 \mathrm{~ms}$ would be associated with improved outcome as manifest by reduced heart failure and mortality when compared to backup ventricular (VVI) pacing at $40 \mathrm{bpm} .{ }^{1}$ The DAVID trial was stopped early because patients randomized to DDDR pacing had a higher frequency of heart failure than the patients receiving conventional therapy. It was quickly appreciated that in patients with underlying cardiac disease and impaired ventricular function that the pacing-induced left bundle branch block conduction pattern created left ventricular contractile dyssynchrony and exacerbated impaired cardiac mechanical function.

In the early 1990s, cardiac resynchronization was introduced with pacing of the epicardial region of the left ventricle via transvenous pacemaker leads positioned in the coronary veins overlying the left ventricle. A series of studies showed that in patients with heart failure and intraventricular conduction delay, cardiac resynchronization therapy involving biventricular pacing of the left and right ventricles improved cardiac hemodynamics and outcome in at-risk cardiac patients. ${ }^{2-5}$ In the recent AHA/ACC/HRS 2008 Guidelines for device-based cardiac therapy, cardiac resynchronization therapy with or without a defibrillator was classified as a class I indication for patients with left ventricular

Address for reprints: Arthur J. Moss, M.D., Heart Research Follow-up Program, Box 653, University of Rochester Medical Center, Rochester, NY14642. Fax: 585-273-5283; E-mail: heartajm@heart.rochester.edu 
ejection fraction $\leq 0.35$, QRS $\geq 120 \mathrm{~ms}$, and New York Heart Association functional class III or ambulatory class IV while receiving optimal medical therapy. ${ }^{6}$ Thus, the selection of patients for resynchronization therapy required the presence of an intraventricular conduction disturbance, frequently of the left bundle branch block type.

In 1990, our Multicenter Automatic Defibrillator Implantation Trial (MADIT) research group initiated a series of randomized device trials that initially focused on the prevention of sudden death in coronary patients with low ejection fractions. Both the MADIT-I and MADIT-II trials showed the lifesaving benefit of the implanted cardioverter defibrillator (ICD) in cardiac patients with a prior myocardial infarction and a low ejection fraction. ${ }^{7,8}$ In secondary analyses from those two trials, patients with a wider QRS complex had an increased risk for heart failure and mortality, a not unexpected finding, with the ICD effective in reducing death even in this high-risk subset. In addition, appropriate ICD firing for ventricular tachycardia and ventricular fibrillation was associated with an increased risk for subsequent heart failure events. ${ }^{9}$ The findings indicated that this increased occurrence of heart failure was due to the myocardial substrate abnormality responsible for the ventricular tachyarrhythmias and not from damage to the myocardium from the delivered ICD shock. In brief, appropriate ICD therapy prevented sudden death, but these saved patients were at increased risk for a later heart failure event, and this finding is most marked in patients with wider QRS complexes.

On the basis of the aforementioned findings, the MADIT Executive Committee initiated in 2004 the randomized MADIT-Cardiac Resynchronization Therapy (MADIT-CRT) trial to determine if prophylactic cardiac resynchronization therapy in combination with a defibrillator (CRT-D) would reduce the occurrence of all-cause mortality or heart failure events, whichever comes first, in cardiac patients with ejection fraction of $\leq 0.30$, QRS duration $\geq 130 \mathrm{~ms}$, and New York Heart Association $\leq$ class II when compared to patients receiving only an implanted defibrillator. ${ }^{10}$ The findings from this trial that involved 1820 patients were recently reported, and CRT-D therapy was associated with a highly significant $34 \%$ reduction in mortality or heart failure events, whichever came first, with similar benefit in patients with ischemic and non- ischemic cardiomyopathy. ${ }^{11}$ Superiority of resynchronization therapy was driven by a $41 \%$ reduction in risk of heart failure events, but especially in patients with QRS duration $\geq 150 \mathrm{~ms}$.

Despite sophisticated technologies involving three-dimension echocardiography and magnetic resonance imaging, the easily and cheaply determined wide QRS complex recorded on the 12-lead ECG is now a key determinant in the selection of at-risk cardiac patients who benefit from cardiac resynchronization therapy.

\section{REFERENCES}

1. Wilkoff BL, Cook JR, Epstein AE, et al. Dual-chamber pacing or ventricular backup pacing in patients with an implantable defibrillator: The Dual Chamber and VVI Implantable Defibrillator (DAVID) Trial. JAMA 2002;288: 3115-3123.

2. Abraham WT, Fisher WG, Smith $\mathrm{AL}_{\text {, }}$ et al. Cardiac resynchronization in chronic heart failure. N Engl J Med 2002 346:1845-1853.

3. Bristow MR, Saxon LA, Boehmer J, et al. Cardiacresynchronization therapy with or without an implantable defibrillator in advanced chronic heart failure. N Engl J Med 2004:350:2140-2150

4. Cleland JG, Daubert JC, Erdmann E, et al. The effect of cardiac resynchronization on morbidity and mortality in heart failure. N Engl J Med 2005;352:1539-1549.

5. Saxon LA, De Marco T, Schafer J, et al. Effects of longterm biventricular stimulation for resynchronization on echocardiographic measures of remodeling. Circulation 2002:105:1304-1310

6. Epstein $\mathrm{AE}$, DiMarco JP, Ellenbogen $\mathrm{KA}$, et al. ACC/ AHA/HRS 2008 Guidelines for Device-Based Therapy of Cardiac Rhythm Abnormalities: A report of the American College of Cardiology/American Heart Association Task Force on Practice Guidelines (Writing Committee to Revise the ACC/AHA/NASPE 2002 Guideline Update for Implantation of Cardiac Pacemakers and Antiarrhythmia Devices): Developed in collaboration with the American Association for Thoracic Surgery and Society of Thoracic Surgeons. Circulation 2008;117:e350-e408.

7. Moss AJ, Hall WJ, Cannom DS, et al. Improved survival with an implanted defibrillator in patients with coronary disease at high risk for ventricular arrhythmia. Multicenter Automatic Defibrillator Implantation Trial Investigators. N Engl J Med 1996;335:1933-1940.

8. Moss AJ, Zareba W, Hall WJ, et al. Prophylactic implantation of a defibrillator in patients with myocardial infarction and reduced ejection fraction. N Engl J Med 2002;346:877883.

9. Goldenberg I, Moss AJ, Hall WJ, et al. Causes and consequences of heart failure after prophylactic implantation of a defibrillator in the multicenter automatic defibrillator implantation trial II. Circulation 2006;113:2810-2817.

10. Moss AJ, Brown MW, Cannom DS, et al. Multicenter automatic defibrillator implantation trial-cardiac resynchronization therapy (MADIT-CRT): Design and clinical protocol. Ann Noninvasive Electrocardiol 2005;10:34-43.

11. Moss AJ, Hall WJ, Cannom DS, et al. Reduction in the risk of heart failure events with preventive cardiac resynchronization therapy. N Engl J Med 2009; (in press). 